Review

\title{
N-type doping strategies for InGaAs
}

\author{
Henry Aldridge Jr, a,*, Aaron G. Lind ${ }^{\mathrm{a}}$, Cory C. Bomberger ${ }^{\mathrm{b}}$, Yevgeniy Puzyrev ${ }^{\mathrm{c}}$, \\ Joshua M.O. Zide ${ }^{\mathrm{b}}$, Sokrates T. Pantelides ${ }^{\mathrm{c}}$, Mark E. Law ${ }^{\mathrm{a}}$, Kevin S. Jones ${ }^{\mathrm{a}}$ \\ a Department of Materials Science and Engineering, University of Florida, Gainesville, FL 32611, USA \\ b Department of Materials Science and Engineering, University of Delaware, Newark, DE 19716, USA \\ ${ }^{\mathrm{c}}$ Department of Physics and Astronomy and Department of Electrical Engineering and Computer Science, Vanderbilt University, Nashville, TN 37235, USA
}

\section{A R T I C L E I N F O}

\section{Keywords:}

III-V semiconductors

Semiconductor processing

Thermal processing

Dopant activation

Dopant diffusion

\begin{abstract}
A B S T R A C T
Significant research effort has been placed into the use of III-V compound semiconductors, including InGaAs as channel materials in CMOS logic devices due to their superior electron mobilities compared to $\mathrm{Si}$ and other more conventional semiconductor materials. One of the major factors preventing industrial adoption of InGaAs as a channel material involves the minimization of source and drain contact resistances. To understand challenges to minimization of contact resistance, this work will outline the effectiveness of several doping approaches that have been attempted for n-type InGaAs including the use of silicon as a dopant and the effectiveness of each approach in achieving the highest level of activation possible. Previous and recently reported dopant diffusion behaviors are also included and discussed.
\end{abstract}

\section{Introduction}

There is continued interest in developing CMOS logic devices with channel materials that allow for faster transistor switching, lower power consumption, and improved heat dissipation than their silicon counterparts. III-V semiconductors, including the ternary alloy $\mathrm{In}_{\mathrm{x}} \mathrm{Ga}_{1-\mathrm{x}} \mathrm{As}$, are currently prime candidates for transistor channel materials due to their superior electron mobilities and injection velocities [1], and are currently included in the ITRS 2.0 roadmap [2]. For this review, the alloy composition lattice-matched to InP $\left(\mathrm{In}_{0.53} \mathrm{Ga}_{0.47} \mathrm{As}\right)$, will be referred to as "InGaAs," unless specifically mentioned. Due to their superior characteristics, III-Vs such as InGaAs will likely be used as a channel material for NMOS transistors, in conjunction with Ge or SiGe based PMOS transistors integrated onto a silicon substrate as demonstrated by multiple groups $[3,4]$.

The need for lower contact resistances is of great importance, since its relative contribution to total device resistance increases with continued device scaling. A source and drain resistivity of less than $15 \Omega-\mu \mathrm{m}$ and $1 \Omega-\mu \mathrm{m}^{2}$ are required to continue current ITRS scaling trends [5]. To attain these goals, maximizing activation of n-type dopants including $\mathrm{Si}$ is a major focus of research. This review discusses the n-type activation challenges of InGaAs and closely related materials as well as strategies to mitigate these limitations. Dopant diffusion behavior is also discussed, as predominant dopants have been recently observed to diffuse significantly during activating anneals.

\section{Processing techniques}

\subsection{Annealing strategies}

Annealing of III-V arsenides is one of the most obvious challenges of processing III-V materials. Group V species tend to evaporate leaving an excess of group III atoms resulting in loss of stoichiometry and degradation of the surface [6,7]. Group V overpressure, proximity capping, and dielectric capping have all been used to prevent the preferential evaporation of group V species [8-12]. Past research suggests that dielectric capping is the most successful method and recent advances with atomic layer deposition have allowed for the creation of thin, dense, and uniform dielectric capping layers to be deposited. Thin capping films better accommodate stress and limit cracks in the encapsulant from mismatches in thermal expansion between the substrate and capping layers. Dense films prevent outdiffusion through pinhole film defects [7]. It is also desirable in most cases to choose capping layers that can be selectively etched and removed from the sample substrate after annealing. Use of a $15 \mathrm{~nm}$ $\mathrm{Al}_{2} \mathrm{O}_{3}$ cap has been demonstrated as an adequate method to protect the surface of InGaAs and related materials [13-15]. Long-term anneals can also lead to growth of non-stoichiometric oxides, which can be mitigated by annealing under an inert gas ambient.

\footnotetext{
* Corresponding author.
} 


\subsection{Material growth conditions}

Growth conditions by MBE or Metal-Organic Chemical Vapor Deposition (MOCVD) have great impact on the material quality and abundance of specific point defects in InGaAs. One method used to influence the relative amount of point defects in III-Vs is by having either an As or $\mathrm{Ga}$ overpressure during growth. Since having $\mathrm{Si}$ occupancy on cation sites is preferred for n-type doping, growing III-Vs such as GaAs and InGaAs with an As overpressure will lower the amount of anion vacancies present in the as-grown material [16] and influence the amount of cation defects that would impact $\mathrm{Si}$ diffusion and activation. Arsenic overpressure is also a central parameter that is accounted for in several DFT and other calculations as a basis for the relative abundance of point defects present in a material [16-19].

\subsection{Doping methods}

A multitude of viable techniques exist to precisely control the doping concentrations in III-V arsenides. Bulk doping of unary and binary semiconductor crystals is often performed during growth from the liquid phase using the Czochralski, liquid encapsulated Czochralski, or Bridgeman growth techniques since they have congruent melting points. Ternary semiconductor systems generally do not have congruent melting points and therefore must be grown via CVD or MBE. Ion implantation has also been a historically useful technique for VLSI processes since it can be self-aligned by lithography features. The earliest doping techniques used an n- or p-type dopant gas or spin-on glass sources that drove the dopant into the bulk of the material by diffusion. Monolayer doping (MLD) is a newer iteration of this process that involves covalently bonding an organic dopant containing molecule onto the surface from a solution before driving the dopant into the bulk with an anneal. The advantages and disadvantages of these doping techniques are discussed for InGaAs in the subsequent sections.

\subsection{Ion implantation}

In ion implantation, dopants are ionized and subsequently accelerated by an electrical potential into the substrate material. The energy and dose of an implant are used to control the depth and concentration respectively of the dopant species in the host substrate. This process is highly energetic with the incident ions often travelling at large fractions of the speed of light. As a result, unlike other techniques, ion implantation results in a large amount of damage ranging from point defects to amorphization at high doses [20].

Ion implantation requires thermal annealing to recover damage and move dopant atoms onto lattice sites. In InGaAs annealing temperatures $>550{ }^{\circ} \mathrm{C}$ are generally required to anneal damage and activate dopants but there is no indication that annealing temperatures greater than $750^{\circ} \mathrm{C}$ improve dopant solubility in these materials [14]. Heated implants have been performed to limit the accumulation of damage during implantation and prevent the formation of amorphous layers in InGaAs at temperatures as low as $80^{\circ} \mathrm{C}$. One side effect of elevated temperature implants into InGaAs can lead to significant dopant channeling, leading to dopant profiles several nanometers deeper for heated implants compared to similar room temperature implants. Electrical activation of dopants implanted at intermediate temperatures $\left(80-100{ }^{\circ} \mathrm{C}\right)$ is improved at short anneal times but prevention of amorphous layer formation in InGaAs is especially important since regrowth of III-V materials is especially disordered relative to $\mathrm{Si}$ [14,21-23]. This problem is exacerbated in the regrowth of 3-D structures as has been shown in $\mathrm{Si}$ and $\mathrm{Ge}[24,25]$. While growth techniques report electrically active doping concretions of $3 \times 10^{19} \mathrm{~cm}^{-3}$ or greater, ion implantation activation saturates around $1.5 \times 10^{19} \mathrm{~cm}^{-3}$ [26].

\subsection{Growth doping}

Epitaxially grown doped layers of InGaAs with CVD or MBE regularly results in the highest $n$-type and p-type doping concentrations in InGaAs and other III-V materials. This seems to be due in large part to the ability to perform growth at temperatures much below the melting temperature of the solid, creating non-equilibrium doping conditions. The process of growing films by MBE is dominated by the surface incorporation kinetics of the precursors rather than towards equilibrium values, which can result in metastable films. A survey of growth doping literature indicates that lower growth temperatures generally result in higher n-type dopant activation [27-33] but some experiments indicate that exceeding the growth temperature during subsequent thermal treatments will result in deactivation in heavily doped substrates. This is likely to occur due to dopant-vacancy complexing $[26,34,35]$. Grown-in doping has a distinct advantage over ion implantation or monolayer doping as it does not require additional annealing steps for dopant incorporation or damage recovery. Multiple studies have shown that high carrier concentrations are often metastable beyond n-type doping concentrations of $1.5 \times 10^{19} \mathrm{~cm}^{-3}$ after subsequent thermal anneals [26,29].

\subsection{Monolayer doping}

Monolayer doping more closely emulates the solid source doping method of the past. In this case, the amount of dopants that can be driven into the bulk are limited by the number of molecules that can be bonded to the surface. It has the potential to create damage-free junctions that are more abrupt than junctions formed with very low energy ion implantation. However, monolayer doping still requires the use of equilibrium diffusion to move dopants into the bulk [36-39]. There is no convincing evidence that monolayer doping provides better electrical activation of n-type dopants than what is observed in ion implantation since it is also limited by dopant-vacancy complexing. Conformal doping of 3-D structures such as fins and nanowires is likely the biggest advantage of monolayer techniques over ion implantation which suffers from shadowing effects.

\section{Dopant selection and activation}

Doping in the III-V family of materials is more varied than in elemental semiconductors since group VI and some group IV dopants behave as n-type dopants in InGaAs. Electrical solubility is one important factor in n-type dopant selection but diffusion behavior is also important to understand in order to develop effective processes for device creation. For sub-10 nm device formation it is generally desired to use dopants that can achieve high activation but also exhibit limited diffusivity.

\subsection{N-type dopants}

Group VI dopants such as S, Se, and Te are obvious choices for ntype dopants but are strongly differentiated by their diffusion characteristics in InGaAs. S is known to be a fast diffuser and likely proceeds by an interstitial mechanism which may complicate the creation of abrupt junctions in InGaAs while Se and Te have exhibited limited diffusion. Group IV species such as $\mathrm{C}, \mathrm{Si}$, Ge and Sn are often referred to as "amphoteric dopants" that can occupy either a group III or group V sublattice site. MBE experiments in GaAs provide the most convincing evidence that group IV dopants are amphoteric, as some experiments have demonstrated $\mathrm{Si}$ as both an $\mathrm{n}$ and p-type dopant. As the ratio of group III to group V flux can be modulated during growth, group IV dopants will preferentially occupy one site over another [4044]. In practice, it is generally observed that these species are nominally $\mathrm{n}$ or $\mathrm{p}$-type in III-V materials when introduced by ion implantation or source diffusion which rely on equilibrium thermal 
processing to introduce dopants onto host lattice sites. Some dopants such as C might be more "amphoteric" in InGaAs depending on stoichiometry with carbon behaving as a n-type donor for In concentration greater than 0.7 and as a p-type acceptor for In concentrations $<0.7[45,46]$.

\subsection{Activation considerations}

$\mathrm{N}$-type doping is generally limited in InGaAs to $0.5-5 \times 10^{19} \mathrm{~cm}^{-3}$ which is considerably lower than the doping levels that can be achieved in elemental $\mathrm{Si}$. Si, Se and Te are perhaps the most heavily studied dopants in InGaAs and despite group IV and group VI dopants occupying different lattice sites, it is observed that all of these dopants result in activation limits of $1.5 \times 10^{19} \mathrm{~cm}^{-3}$ for implanted dopants, which was well summarized in a recent report as shown in Fig. 1 [26]. This discrepancy is perhaps one of the most studied and speculated about aspect of n-type activation in III-V arsenides and has given rise to a number of theories as to what limits dopant activation.

\subsection{Origin of n-type doping limits}

\subsubsection{Chemical solubility limited activation}

Many early experiments attributed the low dopant activation of group VI species in InGaAs to limited chemical solubility such that dopants above the observed electrically active limit were hypothesized to occupy interstitial sites or form clusters. Rutherford Back Scattering (RBS) and Particle Induced X-Ray emission (PIXE) experiments have not shown direct evidence for there being a high number of dopants or clusters occupying interstitial sites in GaAs and instead this conclusion in literature is more often based on electrical results. Other signs of chemical solubility limited activation are noticeably absent from $\mathrm{Si}$ doping in InGaAs. Generally, chemical solubility improves with increasing annealing temperature but recent experiments have shown no temperature dependence on electrical activation of ion implanted Si in InGaAs. Furthermore, n-type dopant activation from MBE and CVD grown InGaAs samples actually improves with decreased growth temperature [26-29,31,32]. Activation limited by chemical solubility would explain differences in dopant activation based on site stoichiometry but it is generally observed that $\mathrm{Si}, \mathrm{Se}$, and $\mathrm{Te}$ all share remarkably similar activation limits [35].

\subsection{Co-implantation}

Even with varying ion implantation conditions, there is ultimately a limit at which further activation is not attained. Co-implantation was pursued in an effort to further decrease the activation ratio of species considered amphoteric in III-Vs. The main principle of this approach is to use one or more additional species to implant into a material. Each of these species would preferentially occupy a certain lattice site, further enhancing or influencing the net activation [47]. One the major recurring details of previous co-implant studies in GaAs is that ion implant energies used were relatively high, ranging from 40 to over $100 \mathrm{keV}$. Others have used isoelectronic co-implants of $\mathrm{Ga}^{+}$or $\mathrm{As}^{+}$with $\mathrm{Si}^{+}$or $\mathrm{Se}^{+}$in an effort to regulate stoichiometry and govern how many anion or cation sites were available for $\mathrm{Si}$ and Se to occupy $[48,49]$. Overall most co-implant studies lead to modest or inconsistent deviations in activation from silicon-only implant cases, and were primarily measured from Hall Effect techniques or sheet numbers [49-53]. Furthermore dopants co-implanted with $\mathrm{Si}$ in InGaAs did not result in active carrier concentrations as high as the sum of their individual electrical solubility limits [54].

\subsection{Amphoteric limited compensation}

Amphoteric limited activation has also been used to explain the large discrepancy between the background chemical concentration and electrically active concentration of group IV dopants. In this model, a n-type group IV dopant preferentially occupies a group III sublattice site up to a certain chemical concentration at which it becomes "amphoteric" and begins to occupy both group III and group V sublattice releasing equal amounts of donors and acceptors and effectively "self-compensating". A subtle variation of this idea is the formation of electrically inactive group IV next-nearest-neighbor neutral pairs $\left(\mathrm{IV}_{\mathrm{III}}-\mathrm{IV}_{\mathrm{V}}\right)$ [55]. In this case, the electrical octet is satisfied and no electrons or holes are released into the bulk.

Many attempts have been made to preferentially modulate the site selection of amphoteric dopants by introducing excess group II, III, V or VI dopants but these experiments generally report limited effectiveness $[47-49,56]$. In the case of co-implantation, the observed increase in activation or amphoteric behavior is generally much less than the coimplant dose.

\subsection{Point defect limited activation}

Point defect limited activation has also been hypothesized as the reason for limited n-type activation of n-type dopants in InGaAs and can consistently explain the observed n-type dopant activation and diffusion behavior in InGaAs. Point defect limited activation predicts that doping limits in InGaAs and other III-V materials will be highly dependent on background doping due to Fermi level effects. Computational studies predict that for heavily n-type III-Vs where the Fermi level shifts towards or into the conduction band, the formation of Frenkel pairs of negatively charged group III vacancies with group III interstitials $\left(\mathrm{V}_{\mathrm{III}}{ }^{3-} \mathrm{I}_{\mathrm{III}}\right)$ become much more favorable $[35,57,58]$. These negatively charged vacancies can then complex with positively charged donors and form electrically compensated pairs such as $\left(\mathrm{V}_{\mathrm{III}}-\mathrm{Si}_{\mathrm{III}}\right)^{2-}$ or $\left(\mathrm{V}_{\mathrm{III}}-\mathrm{S}_{\mathrm{V}}\right)^{2-}$.

Point defect limited activation explains the similar activation levels of both group IV and group VI dopants since compensation will be modulated by the Fermi levels rather than lattice location for a given $n$ type dopant. It also explains the absence of clusters from RBS/PIXE data since this technique would not be sensitive to vacancies $[59,60]$. Furthermore, there is an abundance of experimental evidence that suggest there is a presence of a large number of vacancies in heavily $n$ doped III-V's.

Scanning tunneling Microscopy (STM) has been used to directly observe vacancies in heavily n-type GaAs [61-63] and positron annihilation studies [62,64-70] also generally show that n-type substrates have more vacancies than p-type substrates. A recent study of electrically inactive $\mathrm{P}^{+}$implants into un-doped and heavily $\mathrm{n}$-doped MBE InGaAs have also shown that interstitial loops formed from the implant are much less stable due to interaction with excess vacancies [71]. Growth doped materials also often exhibit lattice superdilation wherein the observed increase in lattice parameter from a given dopant

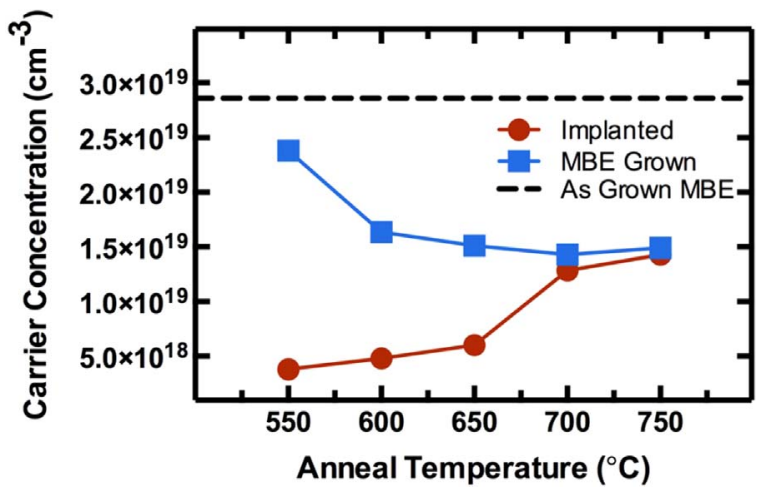

Fig. 1. Calculated net donor concentration $(\mathrm{N})$ of $\mathrm{Si}^{+}$-implanted and $\mathrm{MBE}$ Si-doped $\mathrm{In}_{0.53} \mathrm{Ga}_{0.47} \mathrm{As}$ specimens as a function of annealing temperature after $10 \mathrm{~m}$ furnace anneals at $550,600,650,700$, and $750{ }^{\circ} \mathrm{C}[26]$. 
is much greater than would be expected in simple dopant substitution $[72,73]$. This observation is once again, presumably due to the nucleation of excess vacancies. Metastable n-type doping CVD and MBE InGaAs can also be explained since subsequent thermal treatments beyond the growth result in the formation of dopant defect complexes that could be avoided at lower growth temperatures. This theory also does a much better job of explaining the relevant observations in dopant diffusivity for individual species as well.

\section{Diffusion considerations}

Given the evidence of point defects of significant concentrations present in heavily n-type InGaAs and related materials, it is important to characterize and develop the ability to predict the diffusion of these dopants. This is particularly important for sub-10 $\mathrm{nm}$ devices where a few nanometers of diffusion could have significant effect on operation of the device or neighboring features. This next section discusses dopant behavior of a number of central n-typed dopants in InGaAs.

\subsection{Sulfur diffusion}

$\mathrm{S}$ is known to be a fast a diffuser and is likely an interstitial diffuser in InGaAs, due to the similarity of its concentration profile evolution to known interstitial diffusers like zinc and chromium in GaAs [74]. As shown in Fig. 2, significant portions of the implanted $6 \times 10^{14} \mathrm{~cm}^{-2} \mathrm{~S}^{+}$ dose diffused completely through a $300 \mathrm{~nm}$ InGaAs layer upon annealing. Previously reported diffusivity values of $\mathrm{S}$ in GaAs ranged between $3.0 \times 10^{-15}$ and $5.4 \times 10^{-14} \mathrm{~cm}^{2} \mathrm{~s}^{-1}$ for $\mathrm{S}$ in GaAs annealed at $750{ }^{\circ} \mathrm{C}$ [74-77]. SIMS data from Fig. 2 was used with the Florida object oriented process simulator (FLOOPS) to determine the diffusivity of $\mathrm{S}$ in InGaAs, which found to be $4.3 \times 10^{-13} \mathrm{~cm}^{2} \mathrm{~s}^{-1}$. A Fermi-level dependent diffusion model was implemented for diffusivity extraction [78]. Because of this rapid diffusion, $\mathrm{S}$ is not considered a viable $\mathrm{n}$-type dopant to form shallow junctions in InGaAs for modern devices. More interest has been placed in $\mathrm{Si}$, Se and Te which have all been shown to have limited diffusion in InGaAs and other III-Arsenides.

\subsection{Si diffusion studies}

$\mathrm{Si}$ is a predominant n-type dopant for III-As materials and historically has been reported to have limited diffusivity upon thermal processing $[13,79,80]$. More recent reports have shown that Si diffusion is highly concentration dependent $[81,82]$. Si concentrations above $1-3 \times 10^{-19} \mathrm{~cm}^{-3}$ are shown to diffuse very rapidly while $\mathrm{Si}$ concentrations below this range are generally immobile as shown in Fig. 4b [82]. The point defect-mediated explanation of doping limits in III-V arsenides also provides a sound explanation of the observed diffusivity of $\mathrm{Si}$ in InGaAs as well as other dopants that are less studied in diffusion literature. Fermi-level effects have been shown to modulate the concentration of point defects in III-As semiconductors. The point defect type and concentration play a significant role in mediating $\mathrm{Si}$ diffusion in InGaAs.

Closer comparison of earlier experiments and more recent studies suggest that early reports did not observe the heavy concentration dependence of diffusion because the peak of implanted profiles were below the concentration necessary to result in enhanced diffusion. Experimental and computational studies predict that high n-type doping pushes the Fermi-level towards the conduction band which results in the creation of a large number of cation vacancies. These cation vacancies result in a large enhancement in diffusion for species which diffuse via a group III vacancy mechanism. The enhancement in $\mathrm{Si}$ diffusion due to group III vacancies as a function of Fermi level explains the observed diffusion characteristics of $\mathrm{Si}$ but it also explains the limited diffusivity of Se and Te [83,84]. It is likely that Se and Te which are larger atoms diffuse via a group $\mathrm{V}$ vacancy mechanism. In substrates that are heavily $\mathrm{n}$-doped, the group $\mathrm{V}$ vacancy is not a preferred defect and as a result, the diffusivity of dopants which require group $\mathrm{V}$ vacancies do not show heavily concentration-dependent behavior and exhibit limited diffusivity.

Broadening shoulders of concentration dependent diffusion of ionimplanted and MBE grown silicon in InGaAs are shown in Figs. 3 and 4 respectively. Si marker layers with peak concentrations exceeding $1 \times 10^{20} \mathrm{~cm}^{-3}$ had an $11 \times$ greater effective diffusivity than for the $1 \times 10^{19} \mathrm{~cm}^{-3}$ peak concentration marker layer. The fundamental similarity in advancing profile shape for high concentrations is further evidence that heaver n-type doped regions allow for this rapid diffusion, likely in the form of high concentrations of vacancies that serve as acceptors. Similar concentration-dependent profiles were reported previously for silicon in GaAs systems $[85,86]$.

\subsection{Argon co-implantation effects}

Previous work had employed co-implantation of argon with silicon in GaAs in an effort to enhance activation. This was done in the hope that the Ar would change the stoichiometry such that there is greater availability of cation sites [56]. More recently, co-implantation of InGaAs with other species along with $\mathrm{Si}$, such as $\mathrm{S}, \mathrm{P}$, and $\mathrm{Al}$ was carried out with the purpose of observing if there was any chemical or donor effects leading to greater or reduced activation [54]. Argon was also used as a co-implant, for the purpose of observing a pure damage effect on Si activation. As seen in Fig. 5, there is was a clear shutdown of characteristic shouldering observed in the annealed silicon profiles. This retardation is more prominent with increasing $\mathrm{Ar}^{+}$dose. Other coimplanted species in the study did not inhibit concentration-dependent diffusion of $\mathrm{Si}$ as significantly as Ar with the same implant doses. Calculated net donor concentrations also markedly decreased with increasing argon dose, suggesting that the presence of argon interrupted the silicon activation mechanism as shown in Fig. 6. From these results in comparison to other co-implanted species, it is clear that the effects of co-implanting with argon are more extensive than pure implant damage alone.

It is unlikely that the Argon is pairing with silicon or other dopants to prevent their activation; rather there is likely to be disruption of the lattice due to the generation of inert gas voids, and eventually formation of bubbles as the vacancies coalesce. Bubbles ranging from 3 to $5 \mathrm{~nm}$ in diameter have been observed in GaAs implanted with argon, indicating that it could form in other III-Vs as well [87]. A probable mechanism for this gettering-like behavior is the relieving of interstitial stress of argon atoms in the lattice by being captured by a vacancy. More of this interstitial stress is relieved with the addition of a neighboring vacancy, lowering the overall energy of the system [88]. One possible explanation for the sharp decrease of silicon electrical

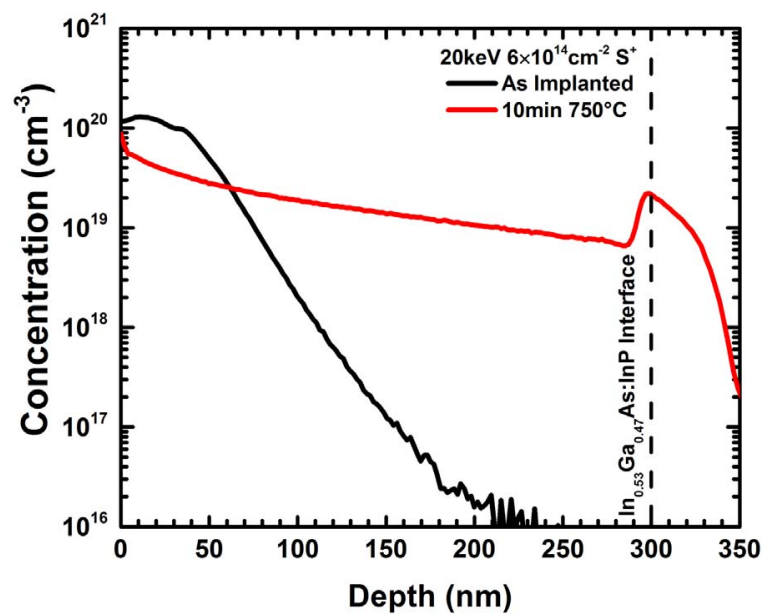

Fig. 2. SIMS profiles of ion-implanted sulfur in InGaAs before and after anneal for $10 \mathrm{~min}$ at $750{ }^{\circ} \mathrm{C}$. 

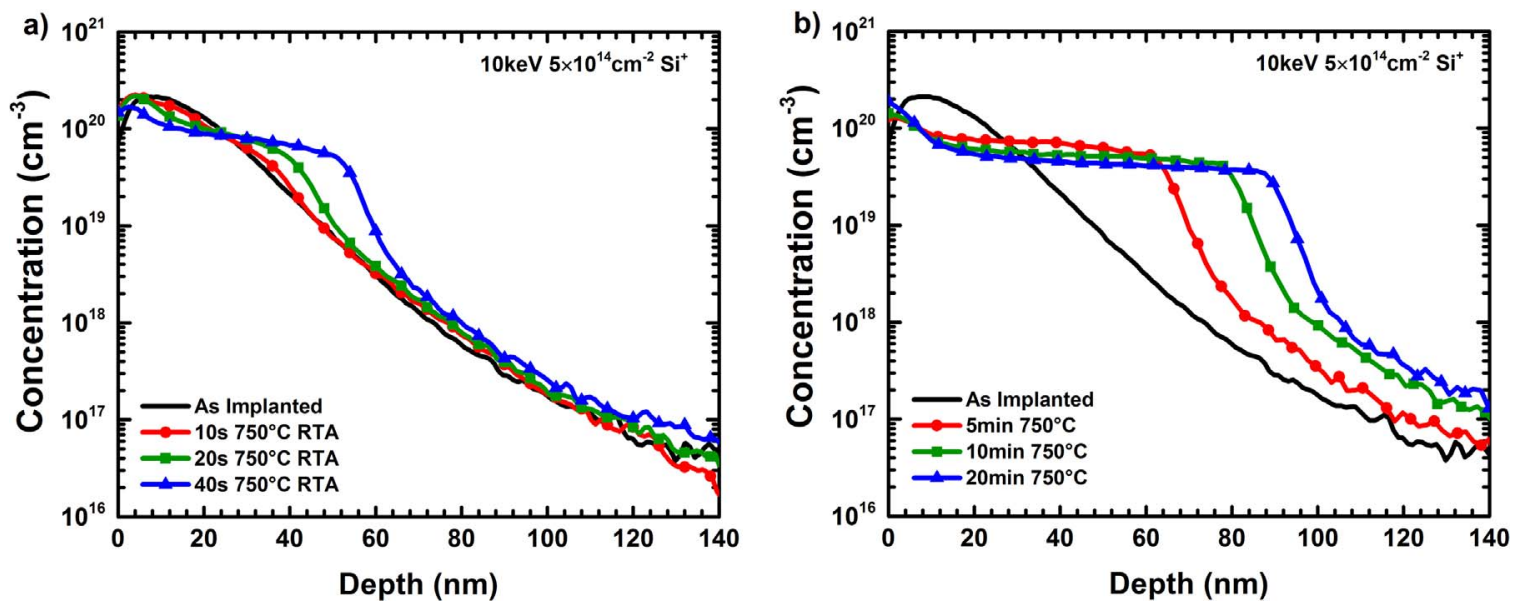

Fig. 3. SIMS profiles of concentration-dependent diffusion observed for ion-implanted InGaAs upon (a) rapid thermal anneal and (b) furnace anneals at $750{ }^{\circ} \mathrm{C}$ [81,102].
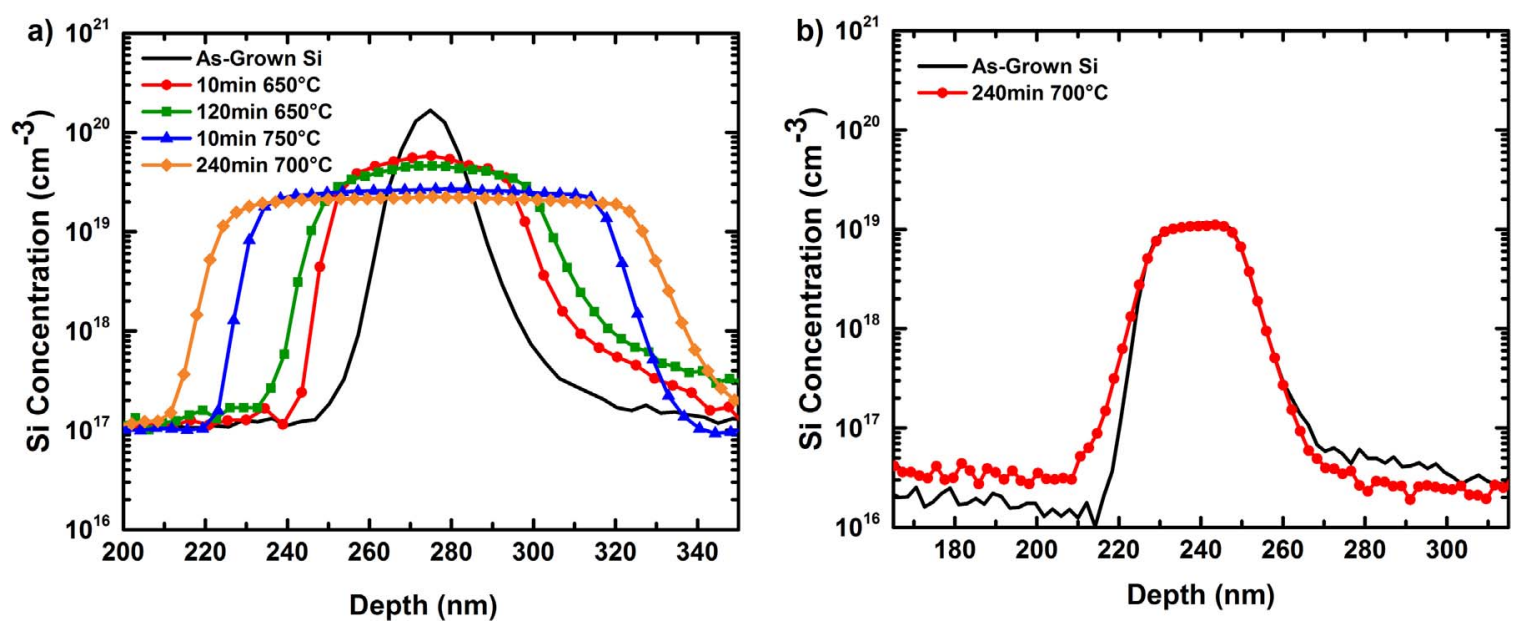

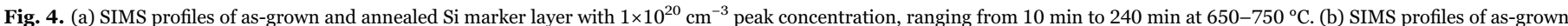
and 240 min $650^{\circ} \mathrm{C}$ annealed Si marker layer with $1 \times 10^{19} \mathrm{~cm}^{-3}$ peak concentration [82].

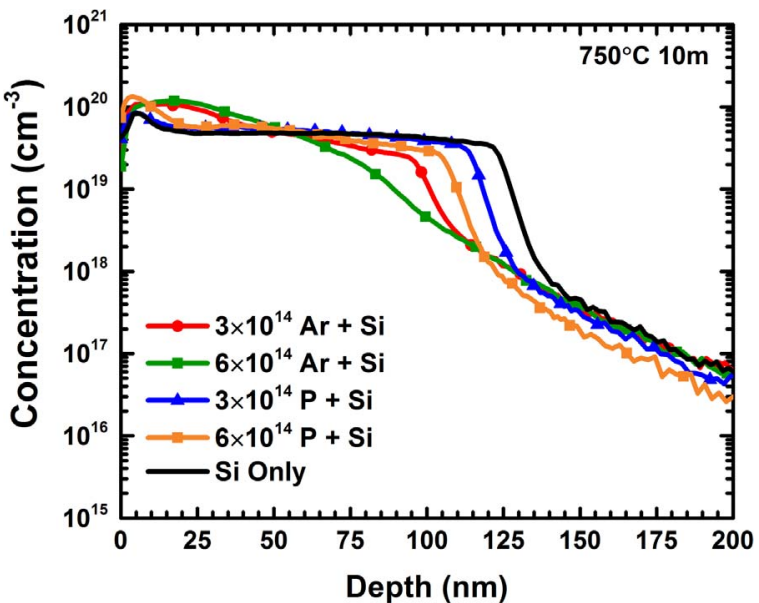

Fig. 5. SIMS profiles of $6 \times 10^{14} \mathrm{~cm}^{-2} \mathrm{Si}^{+}$co-implanted with various doses of $\mathrm{P}^{+}$or $\mathrm{Ar}^{+}$ and annealed for $10 \mathrm{~m} 750{ }^{\circ} \mathrm{C}$. Si diffusion retardation was observed due to increasing co-implant dose.

activation is the known tendency of dislocations to form near inert gas voids, which serve as gettering centers for silicon [89]. There is clear inhibition of both diffusion and activation of $\mathrm{Si}$ in the presence of $\mathrm{Ar}$ in InGaAs, and is likely why implantation of inert gases such as Ar has been used for electrical isolation and gettering centers for both elemental and III-V semiconductors [90].

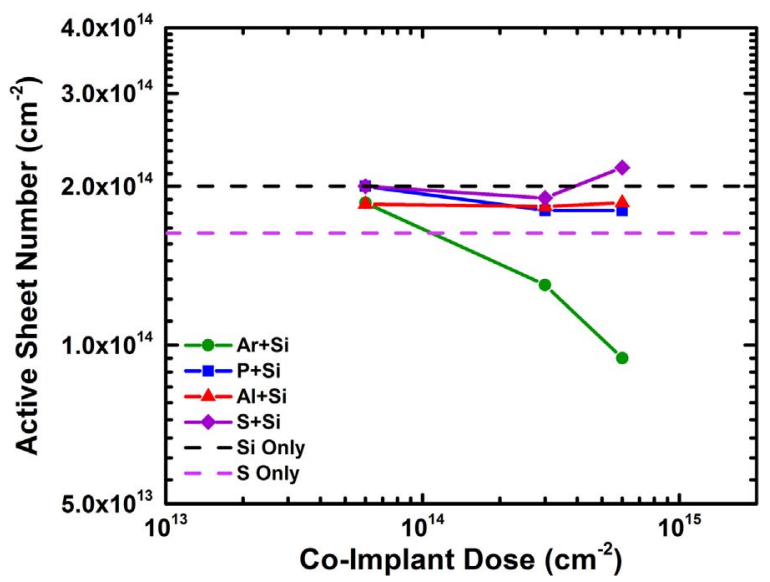

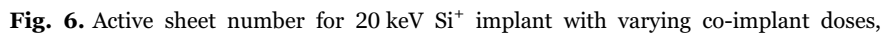
including $\mathrm{Ar}$ in InGaAs, after $10 \mathrm{~m} 750^{\circ} \mathrm{C}$ anneal.

\subsection{DFT calculations of InGaAs system}

Theoretical calculations of defects in InGaAs are less abundant than in silicon, but have been carried out for specific defects of interest, including for the group V sublattice [18,19,91-93]. Despite a comparatively limited volume of literature, a recurring trend from DFT calculations of III-As systems is a pronounced decrease in formation energy of specific defects with an increase in Fermi level. As the Fermi 


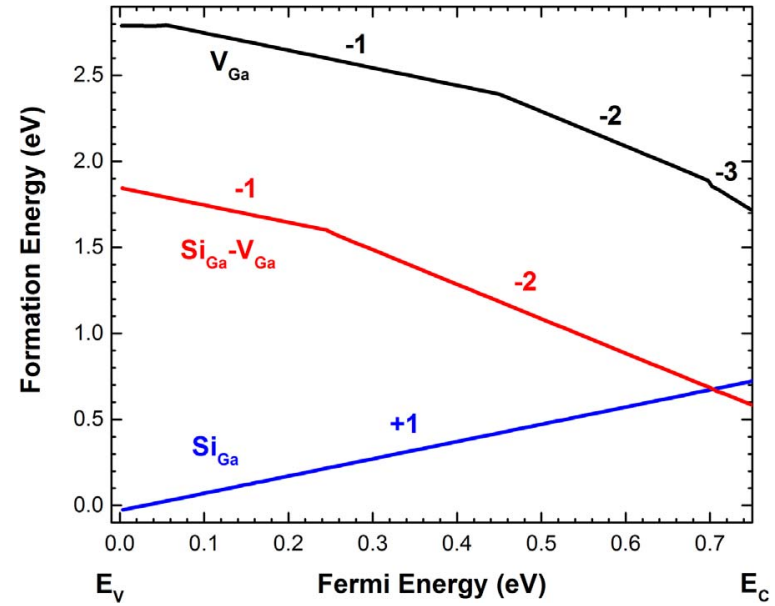

Fig. 7. Calculated formation energies of $\mathrm{V}_{\mathrm{Ga}}, \mathrm{Si}_{\mathrm{Ga}} \mathrm{V}_{\mathrm{Ga}}$ and $\mathrm{Si}_{\mathrm{Ga}}$ with respect to Fermi level in $\mathrm{In}_{0.5} \mathrm{Ga}_{0.5} \mathrm{As}$ system. Values were used in a previously developed diffusion model [82].

level increases toward the conduction band $\mathrm{E}_{\mathrm{c}}$, the formation energy of negatively charged cation vacancies $\mathrm{V}_{\mathrm{III}}^{\mathrm{n}}$ have been calculated to decrease in InGaAs and other III-As materials [18,19,94-97]. A series of DFT calculations were carried out to better characterize the migration barriers and abundance of specific point defects for the $\mathrm{Si}$ doped $\mathrm{In}_{0.53} \mathrm{Ga}_{0.47}$ As system. A second nearest neighbor $\mathrm{Si}_{\mathrm{III}} \mathrm{V}_{\mathrm{III}}$ pair was selected as the primary mechanism for diffusion in InGaAs, for the calculations. Details of the calculations for defect formation energies were included in a previous work, where the values were integrated in a process model for dopant diffusion prediction [82]. As illustrated in Fig. $7, \mathrm{E}_{\mathrm{f}}$ for the $\left(\mathrm{V}_{\mathrm{III}} \mathrm{Si}_{\mathrm{III}}\right)^{2-}$ pair decreases with increasing Fermi level, which is further evidence of the coupled $S i_{I I I}^{+}$and $V_{I I I}^{3-}$ defects serving as the diffusion-aiding mechanism of $\mathrm{Si}$ in InGaAs. Due to the increase in number of negatively charged cation vacancies, silicon diffusion would be enhanced with greater n-type doping, in line with previous experimental reports. The migration barrier for Si diffusion from a second nearest neighbor site was also calculated, and found to be about $2.6 \mathrm{eV}$ for the neutral and doubly negative charged $\mathrm{Si}_{\mathrm{III}}-\mathrm{V}_{\mathrm{III}}$ pair, as shown in Fig. 8.

Other recent DFT studies have been carried out to calculate the energetics of the possible diffusion mechanisms and defects in InGaAs $[98,99]$. Their work supports the trend that formation energies of cation vacancies $\mathrm{V}_{\mathrm{In}} \mathrm{V}_{\mathrm{Ga}}$, decreases with increasing Fermi level and that the formation energy of interstitial $\mathrm{Si}$ is much higher than that of

a)

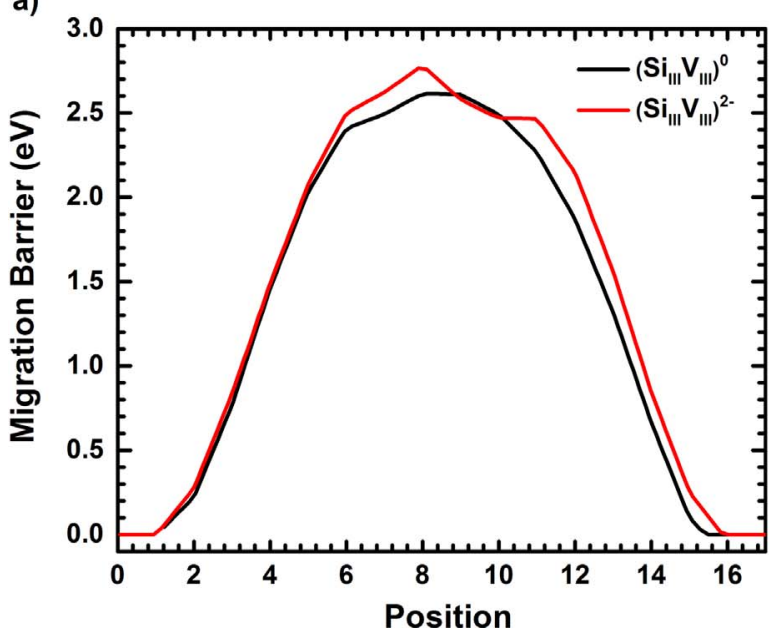

substitutional silicon.

\section{Strategies for maximizing n-type activation of InGaAs}

There are a few central doping methods that were discussed in this work to produce n-type InGaAs. Due to significant amounts of damage introduced through implantation, other doping methods would be preferable to prevent the formation of amorphous regions and subthreshold dislocation loops in the material. Elevated implants ranging from 80 to $300{ }^{\circ} \mathrm{C}$ can be used to prevent amorphization and in turn, poor regrowth of the material is also prevented. Despite using elevated implant temperatures to mitigate implant damage, a post-implantation annealing step is required to reach the highest level of stable dopant activation. Grown-in samples by MBE or MOCVD appear to have promise since activation levels as high as $5 \times 10^{19} \mathrm{~cm}^{-3}$ can be obtained without the requirement of a subsequent annealing step [27,30,84]. Any post-growth thermal processing steps must be kept to a minimum, since the grown-in activation levels decrease towards $\sim 1.5 \times 10^{19} \mathrm{~cm}^{-3}$ with enough thermal processing.

Monolayer doping could hold promise for conformal doping of 3-D structures, and formation of shallow junctions due to the nondestructive nature of the method [36-39]. Similar to ion-implantation, MLD is a method that requires a subsequent thermal processing step to drive in the dopants, although it has the benefit of being a damage free doping method. The amount of annealing must be controlled to prevent excess dopant diffusion and defect mediated compensation, similar to grown-in methods. MLD in III-Vs have been limited to $\mathrm{S}$ and $\mathrm{Si}$ as ntype dopants, and new adsorbates, caps and techniques will be required for the use of additional dopant species.

Silicon diffusion is highly concentration dependent with chemical concentrations exceeding $1-3 \times 10^{19} \mathrm{~cm}^{-3}$, leading to broadening shoulder like profiles with steep junctions. This significant diffusion is observed for both ion-implanted and grown-in samples, indicating that it is not a result of transient enhanced diffusion, but enabled by significant concentrations of cation vacancies in the heavily n-doped regions. Previous works have suggested that regions directly adjacent to heavily Si-doped InGaAs layers could experience a flux of interstitials generated by Frenkel pairs during the formation of compensating vacancies $[100,101]$. Reliable prediction of this diffusion for given thermal treatments is vital for sub-10 nm shallow junction formation.

\subsection{Summary and conclusions}

Ultimately the challenge of maximizing dopant activation in InGaAs

b)

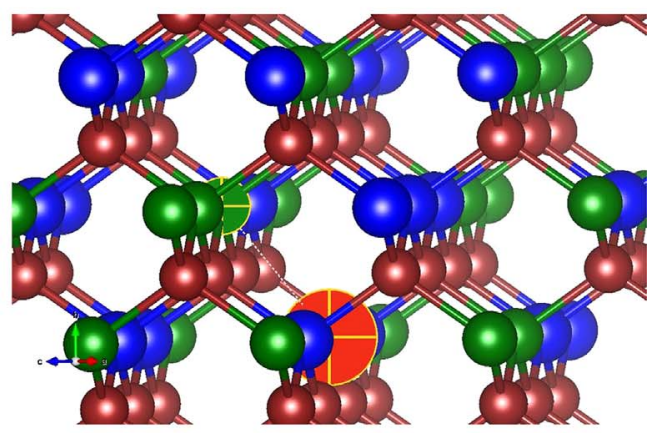

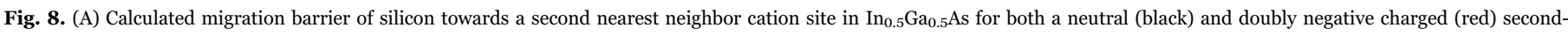

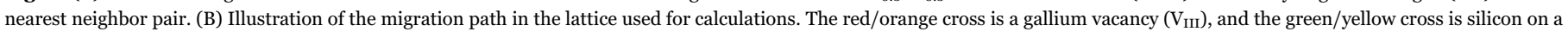
cation site $\left(\mathrm{Si}_{\mathrm{III}}\right)$. (For interpretation of the references to color in this figure legend, the reader is referred to the web version of this article.) 
and related III-Arsenides is non-trivial. There are more variables, and material-specific nuances that can affect the activation and diffusion process. Experimental evidence from previous and recent works suggest that the limits to n-type dopant activation in InGaAs, e.g. silicon, are mediated by compensating point defects in the material, rather than solubility limitations. Coupled with this activation limit, the formation of these point defects can explain the pronounced concentration-dependent $\mathrm{Si}$ diffusion observed in heavily n-type regions of InGaAs. In order to maximize activation of said dopants, low temperature epitaxial doping approaches appear to be the most promising method in cases where there is limited thermal processing following growth. Methods to prevent dopant-vacancy complexing while allowing for activation of dopants would be needed to obtain greater activation, especially in light of additional elevated processing steps.

\section{Acknowledgements}

The authors would like to thank Intel and the Semiconductor Research Corporation for continued support of this work under SRC task\# 2300. C. C. Bomberger is supported by the National Science Foundation (NSF) (DMR-1505574) and by the Delaware Space Grant College and Fellowship Program (NASA Grant-NNX15AI19H). J. M. O. Zide is also supported by the NSF. Work at Vanderbilt was supported by NSF grant ECCS-1508898.

\section{References}

[1] J.A. del Alamo, Nanometre-scale electronics with III-V compound semiconductors, Nature 479 (2011) 317-323. http://dx.doi.org/10.1038/nature10677.

[2] The International Technology Roadmap for Semiconductors 〈http://www.itrs2. net/itrs-news.html).

[3] L. Czornomaz, N. Daix, K. Cheng, D. Caimi, C. Rossel, K. Lister, M. Sousa, J. Fompeyrine, Co-integration of InGaAs n- and SiGe p-MOSFETs into digital CMOS circuits using hybrid dual-channel ETXOI substrates, in: Proceedings of the IEEE International Electron Devices Meeting, 2013, 2.8.1-2.8.4. doi: http://dx.doi.org/ 10.1109/IEDM.2013.6724548

[4] S. Takagi, M. Takenaka, III-V/Ge CMOS technologies on Si platform, in: Proceedings of the Symposium on VLSI Technology, 2010, 147-148. doi: http:// dx.doi.org/10.1109/VLSIT.2010.5556205.

[5] M.J.W. Rodwell, M. Wistey, U. Singisetti, G. Burek, A. Gossard, S. Stemmer, R. Engel-Herbert, Y. Hwang, Y. Zheng, C.V. de Walle, P. Asbeck, Y. Taur, A. Kummel, B. Yu, D. Wang, Y. Yuan, C. Palmstrom, E. Arkun, P. Simmonds, P. McIntyre, J. Harris, M.V. Fischetti, C. Sachs, Technology development \& design for $22 \mathrm{~nm}$ InGaAs/InP-channel MOSFETs, in: Proceedings of the 20th International Conference on Indium Phosphide and Related Materials, 2008, 1-6. doi: http:// dx.doi.org/10.1109/ICIPRM.2008.4703066.

[6] T.E. Haynes, W.K. Chu, T.L. Aselage, S.T. Picraux, Initial decomposition of GaAs during rapid thermal annealing, Appl. Phys. Lett. 49 (1986) 666-668. http:// dx.doi.org/10.1063/1.97562.

[7] T. Haynes, W. Chu, S. Picraux, Direct measurement of evaporation during rapid thermal-processing of capped gaas, Appl. Phys. Lett. 50 (1987) 1071-1073. http://dx.doi.org/10.1063/1.97973.

[8] T. Onuma, T. Hirao, T. Sugawa, Study of encapsulants for annealing Si-implanted GaAs, J. Electrochem. Soc. 129 (1982) 837-840. http://dx.doi.org/10.1149/ 1.2123983.

[9] M. Katayama, Y. Tokuda, Y. Inoue, A. Usami, T. Wada, Ga out-diffusion in rapidthermal-processed GaAs with $\mathrm{SiO}_{2}$ encapsulants, J. Appl. Phys. 69 (1991) 3541-3546. http://dx.doi.org/10.1063/1.348496.

[10] S. Reynolds, D.W. Vook, W.G. Opyd, J.F. Gibbons, Rapid thermal annealing of Siimplanted GaAs with trimethylarsenic overpressure, Appl. Phys. Lett. 51 (1987) 916-918. http://dx.doi.org/10.1063/1.98800.

[11] P.M. Campbell, O. Aina, B.J. Baliga, Prevention of thermal surface damage in GaAs by encapsulated annealing in an arsine ambient, J. Electron. Mater. 15 (1986) 125-131. http://dx.doi.org/10.1007/BF02655325.

[12] J.A. del Alamo, T. Mizutani, Rapid thermal annealing of InP using GaAs and InP proximity caps, J. Appl. Phys. 62 (1987) 3456-3458. http://dx.doi.org/10.1063/ 1.339316.

[13] A. Alian, G. Brammertz, N. Waldron, C. Merckling, G. Hellings, H.C. Lin W.E. Wang, M. Meuris, E. Simoen, K.D. Meyer, M. Heyns, Silicon and selenium implantation and activation in In0.53Ga0.47As under low thermal budget conditions, Microelectron. Eng. 88 (2011) 155-158. http://dx.doi.org/10.1016/ j.mee.2010.10.002.

[14] A.G. Lind, N.G. Rudawski, N.J. Vito, C. Hatem, M.C. Ridgway, R. Hengstebeck B.R. Yates, K.S. Jones, Maximizing electrical activation of ion-implanted Si in In0.53Ga0.47As, Appl. Phys. Lett. 103 (2013) 232102. http://dx.doi.org/ $10.1063 / 1.4835097$.

[15] K.S. Jones, A.G. Lind, C. Hatem, S. Moffatt, M.C. Ridgeway, (Invited) A brief review of doping issues in III-V semiconductors, ECS Trans. 53 (2013) 97-105. http://dx.doi.org/10.1149/05303.0097ecst.

[16] M. Schultz, U. Egger, R. Scholz, O. Breitenstein, U. Gösele, T.Y. Tan, Experimental and computer simulation studies of diffusion mechanisms on the arsenic sublattice of gallium arsenide, J. Appl. Phys. 83 (1998) 5295-5301. http:// dx.doi.org/10.1063/1.367354.

[17] T.Y. Tan, H.-M. You, U.M. Gösele, Thermal equilibrium concentrations and effects of negatively charged Ga vacancies in n-type GaAs, Appl. Phys. A 56 (1993) 249-258. http://dx.doi.org/10.1007/BF00539483.

[18] H.-P. Komsa, A. Pasquarello, Intrinsic defects in GaAs and InGaAs through hybrid functional calculations, Phys. B Condens. Matter 407 (2012) 2833-2837. http:// dx.doi.org/10.1016/j.physb.2011.08.030.

[19] H.-P. Komsa, A. Pasquarello, Comparison of vacancy and antisite defects in GaAs and InGaAs through hybrid functionals, J. Phys. Condens. Matter 24 (2012) 045801. http://dx.doi.org/10.1088/0953-8984/24/4/045801.

[20] K.S. Jones, S. Prussin, E.R. Weber, A systematic analysis of defects in ionimplanted silicon, Appl. Phys. A. 45 (1988) 1-34.

[21] D.K. Sadana, Mechanisms of amorphization and recrystallization in ion implanted III-V compound semiconductors, Nucl. Instrum. Methods Phys. Res. Sect. B Beam Interact. Mater. At. 7 (1985) 375-386. http://dx.doi.org/10.1016/0168583X(85)90585-3.

[22] C. Licoppe, Y.I. Nissim, C. Meriadec, P. Hénoc, Recrystallization kinetics pattern in III-V implanted semiconductors, Appl. Phys. Lett. 50 (1987) 1648-1650. http://dx.doi.org/10.1063/1.97756.

[23] A.G. Lind, M.A. Gill, C. Hatem, K.S. Jones, Electrical activation of ion implanted $\mathrm{Si}$ in amorphous and crystalline In0.53Ga0.47As, Nucl. Instrum. Methods Phys. Res. Sect. B Beam Interact. Mater. At. 337 (2014) 7-10. http://dx.doi.org/ 10.1016/j.nimb.2014.07.009.

[24] R. Duffy, M.J.H.V. Dal, B.J. Pawlak, M. Kaiser, R.G.R. Weemaes, B. Degroote E. Kunnen, E. Altamirano, Solid phase epitaxy versus random nucleation and growth in sub-20 nm wide fin field-effect transistors, Appl. Phys. Lett. 90 (2007) 241912. http://dx.doi.org/10.1063/1.2749186.

[25] R. Duffy, M. Shayesteh, B. McCarthy, A. Blake, M. White, J. Scully, R. Yu, A.M. Kelleher, M. Schmidt, N. Petkov, L. Pelaz, L.A. Marqués, The curious case of thin-body Ge crystallization, Appl. Phys. Lett. 99 (2011) 131910. http:// dx.doi.org/10.1063/1.3643160.

[26] A.G. Lind, H.L. Aldridge, C. Bomberger, C. Hatem, J. Zide, K.S. Jones, Comparison of thermal annealing effects on electrical activation of MBE grown and ion implant Si-doped In0.53Ga0.47As, J. Vac. Sci. Technol. B. 33 (021206) (2015).

[27] T. Fujii, T. Inata, K. Ishii, S. Hiyamizu, Heavily Si-doped InGaAs lattice-matched to InP grown by MBE, Electron. Lett. 22 (1986) 191-192. http://dx.doi.org/ 10.1049/el:19860133.

[28] T. Tsuchiya, H. Nagai, T. Meguro, H. Sakaguchi, Thermal stability of highly Sedoped specular surface of In0.5Ga0.5As grown by low-pressure metalorganic vapor phase epitaxy, J. Cryst. Growth 145 (1994) 403-407. http://dx.doi.org/ 10.1016/0022-0248(94)91083-9.

[29] N. Watanabe, T. Nittono, K. Watanabe, Annealing effect on the carrier concentration in heavily Si-doped n+-InGaAs, Appl. Phys. Lett. 61 (1992) 1945-1947. http://dx.doi.org/10.1063/1.108371.

[30] C.J. Pinzone, N.D. Gerrard, R.D. Dupuis, N.T. Ha, H.S. Luftman, Heavily-doped n-type InP and InGaAs grown by metalorganic chemical vapor deposition using tetraethyltin, J. Appl. Phys. 67 (1990) 6823-6829. http://dx.doi.org/10.1063/ 1.345072

[31] R.A. Kubiak, J.J. Harris, P. Dawson, Electrical and optical properties of Si- and Sn-doped InxGa1-xAs $(\mathrm{x} \approx 0.53)$ grown by molecular beam epitaxy, J. Appl. Phys. 55 (1984) 598-600. http://dx.doi.org/10.1063/1.333072.

[32] Y. Fedoryshyn, M. Beck, P. Kaspar, H. Jaeckel, Characterization of Si volume- and delta-doped InGaAs grown by molecular beam epitaxy, J. Appl. Phys. 107 (2010) 093710. http://dx.doi.org/10.1063/1.3388077.

[33] A.K. Baraskar, M.A. Wistey, V. Jain, U. Singisetti, G. Burek, B.J. Thibeault, Y.J. Lee, A.C. Gossard, M.J.W. Rodwell, Ultralow resistance, nonalloyed ohmic contacts to n-InGaAs, J. Vac. Sci. Technol. B 27 (2009) 2036-2039. http:// dx.doi.org/10.1116/1.3182737.

[34] W. Walukiewicz, Defect formation and diffusion in heavily doped semiconductors, Phys. Rev. B 50 (1994) 5221-5225. http://dx.doi.org/10.1103/ PhysRevB.50.5221.

[35] W. Walukiewicz, Intrinsic limitations to the doping of wide-gap semiconductors, Phys. B Condens. Matter 302-303 (2001) 123-134. http://dx.doi.org/10.1016/ S0921-4526(01)00417-3.

[36] J.C. Ho, R. Yerushalmi, Z.A. Jacobson, Z. Fan, R.L. Alley, A. Javey, Controlled nanoscale doping of semiconductors via molecular monolayers, Nat. Mater. 7 (2008) 62-67. http://dx.doi.org/10.1038/nmat2058.

[37] J.C. Ho, A.C. Ford, Y.-L. Chueh, P.W. Leu, O. Ergen, K. Takei, G. Smith, P. Majhi, J. Bennett, A. Javey, Nanoscale doping of InAs via sulfur monolayers, Appl. Phys. Lett. 95 (2009) 072108. http://dx.doi.org/10.1063/1.3205113.

[38] J.H. Yum, H.S. Shin, R. Hill, J. Oh, H.D. Lee, R.M. Mushinski, T.W. Hudnall, C.W. Bielawski, S.K. Banerjee, W.Y. Loh, W.-E. Wang, P. Kirsch, A study of capping layers for sulfur monolayer doping on III-V junctions, Appl. Phys. Lett. 101 (2012) 253514. http://dx.doi.org/10.1063/1.4772641.

[39] E.Y.J. Kong, P. Guo, X. Gong, B. Liu, Y.C. Yeo, Toward conformal damage-free doping with abrupt ultrashallow junction: formation of Si monolayers and laser anneal as a novel doping technique for InGaAs nMOSFETs, IEEE Trans. Electron Devices 61 (2014) 1039-1046. http://dx.doi.org/10.1109/TED 2014.2306934.

[40] A.Y. Cho, I. Hayashi, P-N junction formation during molecular-beam epitaxy of Ge-doped GaAs, J. Appl. Phys. 42 (1971) 4422-4425. http://dx.doi.org/10.1063/ 
1.1659789.

[41] A.A. Quivy, A.L. Sperandio, E.C.F. da Silva, J.R. Leite, p-Type doping of GaAs(001) layers grown by MBE using silicon as a dopant, J. Cryst. Growth 206 (1999) 171-176.

[42] R. Venkatasubramanian, D.L. Dorsey, K. Mahalingam, Heuristic rules for group IV dopant site selection in III-V compounds, J. Cryst. Growth 175-176 (1997) 224-228.

[43] S.D. Parker, R.L. Williams, R. Droopad, R.A. Stradling, K.W.J. Barnham, S.N. Holmes, J. Laverty, C.C. Phillips, E. Skuras, R. Thomas, X. Zhang, A. StatonBevan, D.W. Pashley, Observation and control of the amphoteric behaviour of Sidoped InSb grown on GaAs by MBE, Semicond. Sci. Technol. 4 (1989) 663. http://dx.doi.org/10.1088/0268-1242/4/8/010.

[44] K. Fujita, A. Shinoda, M. Inai, T. Yamamoto, M. Fujii, D. Lovell, T. Takebe, K. Kobayashi, MBE growth of GaAs p-n junction LEDs on (111)A GaAs substrates using only silicon dopant, J. Cryst. Growth 127 (1993) 50-53.

[45] S.A. Stockman, A.W. Hanson, G.E. Stillman, Growth of carbon-doped p-type InxGa1-xAs $(0<\mathrm{x} \leq 0.53)$ by metalorganic chemical vapor deposition, Appl. Phys. Lett. 60 (1992) 2903-2905. http://dx.doi.org/10.1063/1.106814.

[46] H. Ito, T. Ishibashi, Carbon incorporation in (AlGa)As, (AlIn)As and (GaIn)As ternary alloys grown by molecular beam epitaxy, Jpn. J. Appl. Phys. 30 (1991) L944-L947. http://dx.doi.org/10.1143/JJAP.30.L944.

[47] S. Sugitani, F. Hyuga, K. Yamasaki, Phosphorus coimplantation effects on optimum annealing temperature in Si-implanted GaAs, J. Appl. Phys. 67 (1990) 552-554. http://dx.doi.org/10.1063/1.345245.

[48] A.N. Akimov, L.A. Vlasukova, Y.A. Bumai, K.S. Gorupa, Activation of Si implanted in GaAs at high intensity As co-implantation, Vacuum 63 (2001) 491-494. http:// dx.doi.org/10.1016/S0042-207X(01)00227-5.

[49] T. Ambridge, R. Heckingbottom, E.C. Bell, B.J. Sealy, K.G. Stephens, R.K. Surridge, Effect of dual implants into GaAs, Electron. Lett. 11 (1975) 314. http://dx.doi.org/10.1049/el:19750241.

[50] J.M. Woodcock, Enhancement of the donor activity of implanted selenium in GaAs by gallium implantation, Appl. Phys. Lett. 28 (1976) 226-227. http:// dx.doi.org/10.1063/1.88706.

[51] E.B. Stoneham, G.A. Patterson, J.M. Gladstone, Formation of heavily doped ntype layers in GaAs by multiple ion implantation, J. Electron. Mater. 9, 371383. http://dx.doi.org/10.1007/BF02670855.

[52] T. Inada, S. Kato, T. Ohkubo, T. Hara, Dual species ion implantation in GaAs, Radiat. Eff. 48 (1980) 91-95. http://dx.doi.org/10.1080/00337578008209235.

[53] M.A. Shahid, S. Moffatt, N.J. Barrett, B.J. Sealy, K.E. Puttick, Multiply scanned electron beam annealing of dual implants in GaAs, Radiat. Eff. 70 (1983) 291-299. http://dx.doi.org/10.1080/00337578308219224.

[54] A.G. Lind, H.L. Aldridge Jr, K.S. Jones, C. Hatem, Co-implantation of Al+, P+, and S+ with Si+ implants into In0. 53Ga0. 47As, J. Vac. Sci. Technol. B 33 (2015) 051217. http://dx.doi.org/10.1116/1.4931030.

[55] M.E. Greiner, J.F. Gibbons, Diffusion of silicon in gallium arsenide using rapid thermal processing: experiment and model, Appl. Phys. Lett. 44 (1984) 750-752. http://dx.doi.org/10.1063/1.94904.

[56] S.G. Liu, S.Y. Narayan, C.W. Magee, C.P. Wu, 28Si implantation into 40Ar implant-pretreated semi-insulating GaAs substrates-mobility and activation efficiency enhancement, Appl. Phys. Lett. 41 (1982) 72-75. http://dx.doi.org/ 10.1063/1.93293.

[57] W. Walukiewicz, Amphoteric native defects in semiconductors, Appl. Phys. Lett. 54 (1989) 2094-2096. http://dx.doi.org/10.1063/1.101174.

[58] W. Walukiewicz, Defect formation and diffusion in heavily doped semiconductors, Phys. Rev. B 50 (1994) 5221-5225. http://dx.doi.org/10.1103/ PhysRevB.50.5221.

[59] R.S. Bhattacharya, P.P. Pronko, Damage and lattice location studies of Siimplanted GaAs, Appl. Phys. Lett. 40 (1982) 890-892. http://dx.doi.org/ 10.1063/1.92937.

[60] R.S. Bhattacharya, P.P. Pronko, S.C. Ling, Specific site location of $\mathrm{S}$ and Si in ionimplanted GaAs, Appl. Phys. Lett. 42 (1983) 880-882. http://dx.doi.org/ 10.1063/1.93773.

[61] J. Gebauer, R. Krause-Rehberg, C. Domke, P. Ebert, K. Urban, Identification and quantification of defects in highly Si-doped GaAs by positron annihilation and scanning tunneling microscopy, Phys. Rev. Lett. 78 (1997) 3334-3337. http:// dx.doi.org/10.1103/PhysRevLett.78.3334.

[62] J. Gebauer, E.R. Weber, N.D. Jäger, K. Urban, P. Ebert, Determination of the charge carrier compensation mechanism in Te-doped GaAs by scanning tunneling microscopy, Appl. Phys. Lett. 82 (2003) 2059-2061. http://dx.doi.org/10.1063/ 1.1563835 .

[63] C. Domke, P. Ebert, M. Heinrich, K. Urban, Microscopic identification of the compensation mechanisms in Si-doped GaAs, Phys. Rev. B 54 (1996) 10288-10291. http://dx.doi.org/10.1103/PhysRevB.54.10288.

[64] C. Corbel, M. Stucky, P. Hautojärvi, K. Saarinen, P. Moser, Positron-annihilation spectroscopy of native vacancies in as-grown GaAs, Phys. Rev. B 38 (1988) 8192-8208. http://dx.doi.org/10.1103/PhysRevB.38.8192.

[65] J. Mahony, P. Mascher, Positron-annihilation study of vacancy defects in InAs, Phys. Rev. B 55 (1997) 9637-9641. http://dx.doi.org/10.1103/ PhysRevB.55.9637.

[66] K. Wuyts, G. Langouche, M. Van Rossum, R.E. Silverans, Vacancy-associated Te sites in GaAs, Phys. Rev. B 45 (1992) 6297-6300. http://dx.doi.org/10.1103/ PhysRevB.45.6297.

[67] S. Dannefaer, B. Hogg, D. Kerr, Investigation of defects in gallium arsenide using positron annihilation, Phys. Rev. B 30 (1984) 3355-3366. http://dx.doi.org/ 10.1103/PhysRevB.30.3355.

[68] J.-L. Lee, L. Wei, S. Tanigawa, M. Kawabe, Impurity effect on the creation of Ga vacancies in a Si-doped layer grown on Be-doped GaAs by molecular-beam epitaxy, J. Appl. Phys. 68 (1990) 5571-5575. http://dx.doi.org/10.1063/ 1.346992.

[69] J.-L. Lee, A. Uedono, S. Tanigawa, J.Y. Lee, Vacancy-type defects in Si+-implanted GaAs and its effects on electrical activation by rapid thermal annealing, J. Appl. Phys. 67 (1990) 6153-6158. http://dx.doi.org/10.1063/1.345177.

[70] J.-L. Lee, L. Wei, S. Tanigawa, M. Kawabe, Effects of impurities Si and Be on the creation of $\mathrm{Ga}$ vacancies and $\mathrm{Ga}$ interstitials in GaAs grown by molecular beam epitaxy, Appl. Phys. Lett. 58 (1991) 1524-1526. http://dx.doi.org/10.1063/ 1.105166.

[71] A.G. Lind, H.L. Aldridge, C.C. Bomberger, C. Hatem, J.M.O. Zide, K.S. Jones, Fermi-level effects on extended defect evolution in $\mathrm{Si}+$ and $\mathrm{P}+$ implanted In0.53Ga0.47As, ECS J. Solid State Sci. Technol. 5 (2016) P3073-P3077. http:// dx.doi.org/10.1149/2.0141604jss.

[72] D.T.J. Hurle, A thermodynamic analysis of native point defect and dopant solubilities in zinc-blende III-V semiconductors, J. Appl. Phys. 107 (2010) 121301. http://dx.doi.org/10.1063/1.3386412.

[73] J.B. Mullin, B.W. Straughan, C.M.H. Driscoll, A.F.W. Willoughby, Lattice superdilation phenomena in doped GaAs, J. Appl. Phys. 47 (1976) 2584-2587. http:// dx.doi.org/10.1063/1.322977.

[74] M. Uematsu, P. Werner, M. Schultz, T.Y. Tan, U.M. Gösele, Sulfur diffusion and the interstitial contribution to arsenic self-diffusion in GaAs, Appl. Phys. Lett. 67 (1995) 2863-2865. http://dx.doi.org/10.1063/1.114810.

[75] H. Matino, Reproducible sulfur diffusion into GaAs, Solid-State Electron. 17 (1974) 35-39. http://dx.doi.org/10.1016/0038-1101(74)90111-7.

[76] A.B.Y. Young, G.L. Pearson, Diffusion of sulfur in gallium phosphide and gallium arsenide, J. Phys. Chem. Solids 31 (1970) 517-527. http://dx.doi.org/10.1016/ 0022-3697(70)90092-2.

[77] B. Goldstein, Diffusion in compound semiconductors, Phys. Rev. 121 (1961) 1305-1311. http://dx.doi.org/10.1103/PhysRev.121.1305.

[78] M.E. Law, FLOODS/FLOOPS Manual, Univ. Florida, Gainesville, FL, 1993 (http://www.flooxs.ece.ufledu).

[79] E. Hailemariam, S.J. Pearton, W.S. Hobson, H.S. Luftman, A.P. Perley, Doping of In0.53Ga0.47As and In0.52Al0.48As by $\mathrm{Si}+$ and $\mathrm{Be}+$ ion implantation, J. Appl. Phys. 71 (1992) 215-220. http://dx.doi.org/10.1063/1.350745.

[80] M.V. Rao, S.M. Gulwadi, P.E. Thompson, A. Fathimulla, O.A. Aina, Halogen lamp rapid thermal annealing of Si- and Be-implanted In0.53Ga0.47As, J. Electron. Mater. 18 (1989) 131-136. http://dx.doi.org/10.1007/BF02657398.

[81] H.L. Aldridge, A.G. Lind, M.E. Law, C. Hatem, K.S. Jones, Concentrationdependent diffusion of ion-implanted silicon in In0.53Ga0.47As, Appl. Phys. Lett. 105 (2014) 042113. http://dx.doi.org/10.1063/1.4892079.

[82] H.L. Aldridge Jr, A.G. Lind, C.C. Bomberger, Y. Puzyrev, C. Hatem, S. Pantelides, M.E. Law, K.S. Jones, Implantation and diffusion of silicon marker layers in In0.53Ga0.47As, J. Electron. Mater. 45 (2016) 4282-4287. http://dx.doi.org/ 10.1007/s11664-016-4616-0.

[83] S.J. Pearton, A.R.V. Neida, J.M. Brown, K.T. Short, L.J. Oster, U.K. Chakrabarti, Ion implantation damage and annealing in InAs, GaSb, and GaP, J. Appl. Phys. 64 (1988) 629-636. http://dx.doi.org/10.1063/1.341952.

[84] T. Orzali, A. Vert, R.T.P. Lee, A. Norvilas, G. Huang, J.L. Herman, R.J.W. Hill, S.S.P. Rao, Heavily tellurium doped n-type InGaAs grown by MOCVD on $300 \mathrm{~mm}$ Si wafers, J. Cryst. Growth 426 (2015) 243-247. http://dx.doi.org/10.1016/ j.jcrysgro.2015.05.007.

[85] C.C. Lee, M.D. Deal, K.S. Jones, H.G. Robinson, J.C. Bravman, Effect of ion energy on the diffusion of Si implanted into GaAs, J. Electrochem. Soc. 141 (1994) 2245-2249. http://dx.doi.org/10.1149/1.2055097.

[86] T. Ahlgren, J. Likonen, J. Slotte, J. Räisänen, M. Rajatora, J. Keinonen, Concentration dependent and independent Si diffusion in ion-implanted GaAs, Phys. Rev. B 56 (1997) 4597-4603. http://dx.doi.org/10.1103/ PhysRevB.56.4597.

[87] W. Walukiewicz, Z. Liliental-Weber, J. Jasinski, M. Almonte, A. Prasad, E.E. Haller, E.R. Weber, P. Grenier, J.F. Whitaker, High resistivity and ultrafast carrier lifetime in argon implanted GaAs, Appl. Phys. Lett. 69 (1996) 2569-2571. http://dx.doi.org/10.1063/1.117702.

[88] M.J. Norgett, A.B. Lidiard, The migration of inert gases in ionic crystals, Philos Mag. 18 (1968) 1193-1210. http://dx.doi.org/10.1080/14786436808227750.

[89] D.M. Follstaedt, S.M. Myers, J.C. Barbour, G.A. Petersen, J.L. Reno, L.R. Dawson, S.R. Lee, Formation of cavities in GaAs and InGaAs, Nucl. Instrum. Methods Phys. Res. Sect. B Beam Interact. Mater. At. 160 (2000) 476-498. http://dx.doi.org/ 10.1016/S0168-583X(99)00618-7.

[90] S.J. Pearton, Ion implantation for isolation of III-V semiconductors, Mater. Sci. Rep. 4 (1990) 313-363. http://dx.doi.org/10.1016/S0920-2307(05)80001-5.

[91] S.T. Murphy, A. Chroneos, R.W. Grimes, C. Jiang, U. Schwingenschlögl, Phase stability and the arsenic vacancy defect in $\operatorname{In}_{x} \mathrm{Ga}_{1-\mathrm{x}} \mathrm{As}$, Phys. Rev. B 84 (2011) 184108. http://dx.doi.org/10.1103/PhysRevB.84.184108.

[92] T.V. Bezyazychnaya, M.V. Bogdanovich, A.V. Grigor'ev, V.M. Zelenkovskii, V.V. Kabanov, D.M. Kabanov, Y.V. Lebiadok, A.G. Ryabtsev, G.I. Ryabtsev, M.A. Shchemelev, Point defects and amplification in active layers of InGaAs/ AlGaAs heterostructures, Phys. Solid State 55 (2013) 2165-2168. http:// dx.doi.org/10.1134/S1063783413100041.

[93] A.A. Bonapasta, P. Giannozzi, Effects of strain and local charge on the formation of deep defects in III-V ternary alloys, Phys. Rev. Lett. 84 (2000) 3923-3926. http://dx.doi.org/10.1103/PhysRevLett.84.3923.

[94] J.E. Northrup, S.B. Zhang, Dopant and defect energetics: Si in GaAs, Phys. Rev. B 47 (1993) 6791-6794. http://dx.doi.org/10.1103/PhysRevB.47.6791.

[95] S.B. Zhang, J.E. Northrup, Chemical potential dependence of defect formation energies in GaAs: application to Ga self-diffusion, Phys. Rev. Lett. 67 (1991) 
2339-2342. http://dx.doi.org/10.1103/PhysRevLett.67.2339.

[96] G.A. Baraff, M. Schlüter, Electronic structure, total energies, and abundances of the elementary point defects in GaAs, Phys. Rev. Lett. 55 (1985) 1327-1330. http://dx.doi.org/10.1103/PhysRevLett.55.1327.

[97] H.A. Tahini, A. Chroneos, S.T. Murphy, U. Schwingenschlögl, R.W. Grimes, Vacancies and defect levels in III-V semiconductors, J. Appl. Phys. 114 (2013) 063517. http://dx.doi.org/10.1063/1.4818484.

[98] C.-W. Lee, B. Lukose, M.O. Thompson, P. Clancy, Energetics of neutral Si dopants in InGaAs: an ab initio and semiempirical Tersoff model study, Phys. Rev. B 91 (2015) 094108. http://dx.doi.org/10.1103/PhysRevB.91.094108.

[99] S.R. Lee, A.F. Wright, N.A. Modine, C.C. Battaile, S.M. Foiles, J.C. Thomas, A. Van der Ven, First-principles survey of the structure, formation energies, and transi- tion levels of As-interstitial defects in InGaAs, Phys. Rev. B 92 (2015) 045205.

http://dx.doi.org/10.1103/PhysRevB.92.045205.

[100] T. Takamoto, M. Yumaguchi, E. Ikeda, T. Agui, H. Kurita, M. Al-Jassim, Mechanism of $\mathrm{Zn}$ and Si diffusion from a highly doped tunnel junction for $\mathrm{InGaP} /$ GaAs tandem solar cells, J. Appl. Phys. 85 (1999) 1481-1486. http://dx.doi.org/ $10.1063 / 1.369278$.

[101] H. Fushimi, M. Shinohara, K. Wada, Effects of native defects on carrier concentrations in heavily Si-doped and adjoining lightly doped GaAs layers, J. Appl. Phys. 81 (1997) 1745-1751. http://dx.doi.org/10.1063/1.364030.

[102] H.L. Aldridge, A.G. Lind, M.E. Law, C. Hatem, K.S. Jones, Continuum modelling of silicon diffusion and activation in in $0.53 \mathrm{Ga} 0.47 \mathrm{As}$, ECS Trans. 66 (2015) 57-61. http://dx.doi.org/10.1149/06607.0057ecst. 